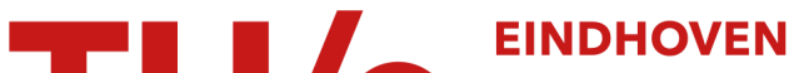 \\ UNIVERSITY OF \\ TECHNOLOGY
}

\section{Extremely Large Magnetoresistance in Boron-Doped Silicon}

\section{Citation for published version (APA):}

Schoonus, J. J. H. M., Bloom, F. L., Wagemans, W., Swagten, H. J. M., \& Koopmans, B. (2008). Extremely Large Magnetoresistance in Boron-Doped Silicon. Physical Review Letters, 100(12), 127202-1/4. [127202]. https://doi.org/10.1103/PhysRevLett.100.127202

DOI:

10.1103/PhysRevLett.100.127202

Document status and date:

Published: 01/01/2008

\section{Document Version:}

Publisher's PDF, also known as Version of Record (includes final page, issue and volume numbers)

\section{Please check the document version of this publication:}

- A submitted manuscript is the version of the article upon submission and before peer-review. There can be important differences between the submitted version and the official published version of record. People interested in the research are advised to contact the author for the final version of the publication, or visit the $\mathrm{DOI}$ to the publisher's website.

- The final author version and the galley proof are versions of the publication after peer review.

- The final published version features the final layout of the paper including the volume, issue and page numbers.

Link to publication

\section{General rights}

Copyright and moral rights for the publications made accessible in the public portal are retained by the authors and/or other copyright owners and it is a condition of accessing publications that users recognise and abide by the legal requirements associated with these rights.

- Users may download and print one copy of any publication from the public portal for the purpose of private study or research.

- You may not further distribute the material or use it for any profit-making activity or commercial gain

- You may freely distribute the URL identifying the publication in the public portal.

If the publication is distributed under the terms of Article 25fa of the Dutch Copyright Act, indicated by the "Taverne" license above, please follow below link for the End User Agreement:

www.tue.nl/taverne

Take down policy

If you believe that this document breaches copyright please contact us at:

openaccess@tue.nl

providing details and we will investigate your claim. 


\title{
Extremely Large Magnetoresistance in Boron-Doped Silicon
}

\author{
J. J. H. M. Schoonus, F. L. Bloom, W. Wagemans, H. J. M. Swagten, and B. Koopmans \\ Department of Applied Physics, cNM, Eindhoven University of Technology, The Netherlands
}

(Received 21 August 2007; published 27 March 2008)

\begin{abstract}
Boron-doped $\mathrm{Si}_{-} \mathrm{SiO}_{2}-\mathrm{Al}$ structures are fabricated to study extremely large magnetoresistance (MR) effects. Current-voltage characteristics show a nonlinear behavior, dominated by an autocatalytic process of impact ionization. At low temperatures, the magnetic field postpones the onset of impact ionization to higher electric fields. This results in a symmetric positive MR of over $10000 \%$ at $400 \mathrm{kA} / \mathrm{m}$. Applying a magnetic field leads to an increase of the acceptor level compared to the valence band as deduced by admittance spectroscopy. A macroscopic transport model is introduced to describe how the MR is controlled by voltage, electrode spacing, and oxide thickness.
\end{abstract}

Research in magnetotransport of semiconductors without using magnetic materials [1,2] is a field of ongoing interest. Silicon holds exceptional promise for magnetoelectronics [3], by virtue of its long spin coherence [4] and compatibility with the current CMOS technology. Within the past decades the influence of the magnetic field on p-type silicon devices has been explored as well. Negative magnetoresistance (MR) in the impurity conduction regime at microwave frequencies [5], in the hopping regime [6], and positive $\mathrm{MR}$ in $\mathrm{FeSi}_{-} \mathrm{SiO}_{2}-\mathrm{Si}$ junctions in the vicinity of a resistive transition [7] have been reported. Also the effect of a magnetic field on the metal-insulator transition in two-dimension electron systems [8] has been investigated. However, the maximum MR observed up to now was only $40 \%$ at typical fields of $1 \mathrm{~T}$. Although the nonlinear electrical transport in metal-oxidesemiconductor heterointerfaces has been investigated thoroughly [9], there still is a lack of consensus about the origin of the mechanism causing this MR. Recently, it was shown that in semi-insulating $\mathrm{GaAs} / i-\mathrm{GaAs} / \mathrm{Au}$, the resistance increases by more than 3 orders of magnitude at fields of $1 \mathrm{~T}$ over a broad temperature range, and is tentatively ascribed to a magnetic-field dependent avalanche breakdown phenomenon [10]. Observation as well as a comprehensive explanation of larger MR in silicon, based on this phenomenon, will be an important progress towards new spintronics applications.

In this Letter, we show for the first time in boron-doped silicon a robust MR of well above $10000 \%$, which is in contrast to other reports on silicon significantly larger at much smaller magnetic fields. By systematic investigation of the role of the thin silicon dioxide layer used to inject the carriers in the silicon (equivalent to the Schottky barrier in Ref. [10]), we establish the key ingredient for the understanding of the enhanced magnetoresistance effect; the tunnel injection provides the required energy to trigger a transition to a high mobility transport regime by an autocatalytic process of impact ionization. A magnetic field raises the acceptor level as described by Sladek [11], and previously reported for GaAs [12], by which the activation energy for the autocatalytic process of impact ionization exponentially increases, and thereby strongly suppresses the current.

The $\mathrm{Si}: \mathrm{B} / \mathrm{SiO}_{2} / \mathrm{Al}$ lateral devices were fabricated from a $500 \mu \mathrm{m}$ thick boron-doped silicon wafer $(15 \Omega \mathrm{cm}$, $p$-type $1 \times 10^{15} \mathrm{~cm}^{-3}$ ) produced by Shin-Etsu Handotai Group. The mobility has been measured as $3.4 \times$ $10^{2} \mathrm{~cm}^{2} / \mathrm{V} \mathrm{s}$ at room temperature. On top of the native surface oxide layer two aluminum electrodes of $100 \mu \mathrm{m}$ wide, separated $50 \mu \mathrm{m}$ from each other, are deposited by dc magnetron sputtering. As shown in Fig. 1, the magnetic field, aligned parallel to the plane of the substrate and swept from -400 to $400 \mathrm{kA} / \mathrm{m}$, decreases the current by 2 orders of magnitude at $4 \mathrm{~K}$ and a bias voltage of $20 \mathrm{~V}$. The corresponding MR, defined as $(R(H) / R(0)-1) \times$ $100 \%$, with $R(0)$ and $R(H)$ the resistances at zero and applied field, respectively, is shown on the right axis. The MR sharply increases for fields up to around $100 \mathrm{kA} / \mathrm{m}$, whereafter it continuously increases to a value of $10000 \%$ at $400 \mathrm{kA} / \mathrm{m}$, mostly never reaching saturation. To further explore these effects, we have varied the angle between current and magnetic field, and found no pronounced anisotropy in the MR. Identically prepared devices with Si:B wafers from two different suppliers, ITME $(1-30 \Omega \mathrm{cm})$

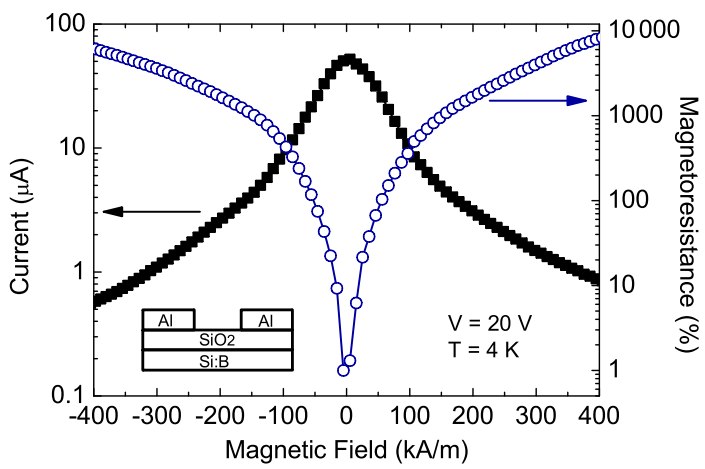

FIG. 1 (color online). Current as function of the magnetic field (left axis) and corresponding magnetoresistance (right axis) for $\mathrm{Si}: \mathrm{B}(15 \Omega \mathrm{cm})$ with native oxide. 
and Si-Mat (3-9 $\Omega \mathrm{cm}$ ), showed similar behavior with reproducible resistance changes up to 8 orders of magnitude. This demonstrates that the effect is robust and the corresponding mechanism should be related to intrinsic transport processes in these devices.

The extremely large MR effect shown in Fig. 1 can be directly related to the highly nonlinear current-voltage behavior, which is observed for various magnetic fields (see Fig. 2). In the inset, a log-linear plot of the same data is given. The voltage is swept in the positive direction and at the threshold voltage (i.e., $7 \mathrm{~V}$ ) a sharp increase in current by several orders of magnitude is observed. An applied magnetic field postpones this threshold voltage to higher electric fields and the curves become less and less steep. As suggested by Sun et al. [10], the sharp increase in current can be interpreted as an autocatalytic process of impact ionization of the shallow acceptor boron, and the threshold voltage is determined by the relative location of the Fermi level in thermal equilibrium and of that of the acceptor level. A hysteric behavior, small compared to the threshold voltage, is observed when the voltage is gradually lowered under the threshold voltage, indicating that the autocatalytic process of impact ionization can be sustained at a lower bias voltage. This is a well-known principle from breakdown simulations with impact ionization models in MOSFETs [13].

To further identify the role of the acceptor ionization for the observed MR, Fig. 3(a) presents the temperature dependence of the $I-V$ curves at 0 and $400 \mathrm{kA} / \mathrm{m}$. With increasing temperature, the resistivity decreases as also explicitly displayed in the inset, and the onset of impact ionization occurs at lower bias voltages. Simultaneously, the MR collapses to relatively small values ( $\sim 14 \%$ at $36 \mathrm{~K})$ due to two reasons. At temperatures in the range of $20-$ $30 \mathrm{~K}$, the reduction is due to an effect of the magnetic field on the impact ionization, as will be clarified further on in this Letter. Starting from around $100 \mathrm{~K}$, thermal ionization of impurities into the high mobility valence band is completed [see inset Fig. 3(a)], even further quenching the MR.

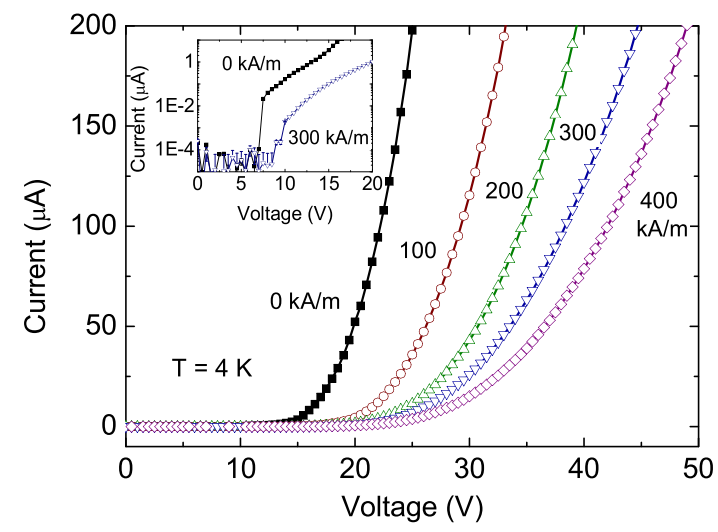

FIG. 2 (color online). $\quad I-V$ curves for various applied magnetic fields; the inset shows the same data plotted on a log-linear scale.
Based on these findings, a possible mechanism governing this magnetoresistance effect is quantization of the carrier motion by the magnetic field, as described by Sladek for $n$-InSb [11]. For small enough impurity concentrations, the acceptor wave functions are centered on the acceptor atoms, but they still have a finite overlap. The magnetic field causes shrinkage of the acceptor wave functions, proportional to the effective mass. The hole orbitals become more localized in the vicinity of the acceptor ions and the overlap by the tails is reduced. By reducing the overlap of the wave functions, the field gradually narrows the impurity band and increases it to a higher energy compared to the valence band, causing the carriers to freeze out and localize on the impurity ions, resulting in a high resistivity.

To confirm this speculation, admittance as a function of temperature was measured to determine the energy of the acceptor levels $E_{a}$ [14]. In thermal equilibrium and at zero bias, a peak in the conductance and step in the capacitance graph are observed at the temperature at which the acceptor ionizes [Fig. 3(b)]. For frequencies $(\omega / 2 \pi)$ within the range of 10 and $100 \mathrm{kHz}$, and for fields of 0 and $400 \mathrm{kA} / \mathrm{m}$, the temperatures at the inflection points of the capacitance curves $T_{i}$ are extracted. A plot of $\omega / T_{i}^{3 / 2}$ vs $1 / T_{i}$ [Fig. 3(c)] yields a straight line with a slope corresponding to the acceptor energy with respect to the valence band. The intercept where $1 / T_{i}=0$ is equal to $9.7 \times 10^{15} m_{e}^{* 3 / 2} C_{p} \beta$ [14], with $C_{p}$ the capture coefficient,
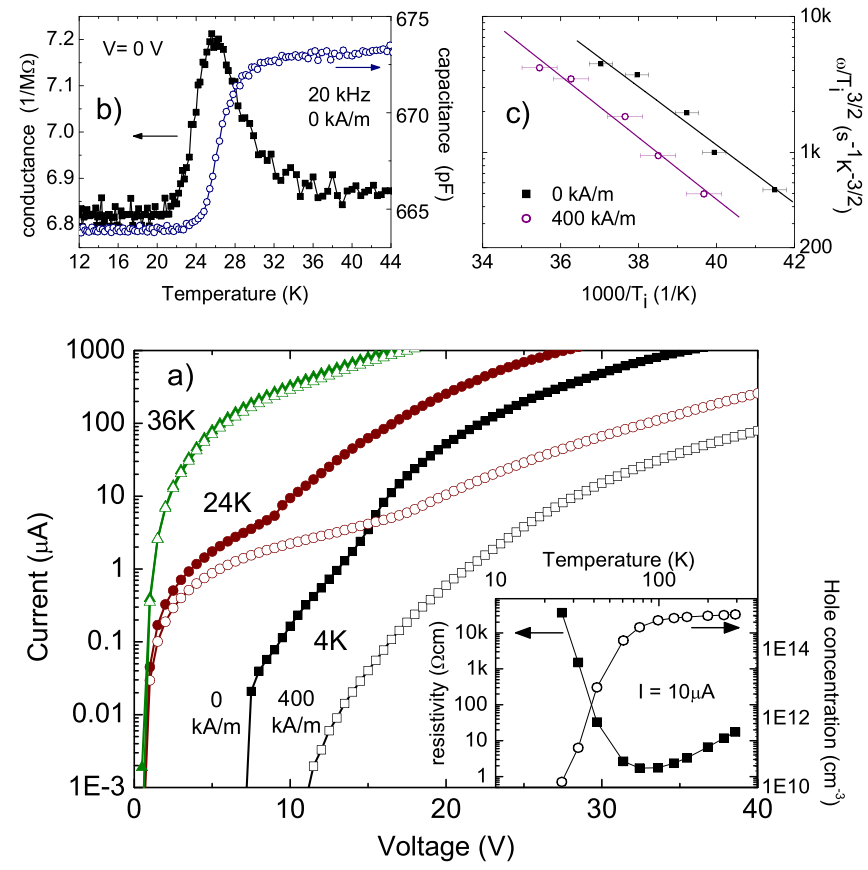

FIG. 3 (color online). (a) $I-V$ curves at $0 \mathrm{kA} / \mathrm{m}$ (filled) and $400 \mathrm{k} / \mathrm{Am}$ (open) at various temperatures, (inset) resistivity and hole concentration as function of temperature, (b) conductance and capacitance versus temperature and (c) plot of $\omega / T_{i}^{3 / 2}$ versus reciprocal peak temperature for 0 and $400 \mathrm{kA} / \mathrm{m}$. 
which is the rate at which ionized acceptors capture holes, and where an effective mass of $m_{e}^{*}=0.5$ and degeneracy $\beta=4$ can be used. In zero field, we deduced for the acceptor energy $(43.9 \pm 0.2) \mathrm{meV}$. This is in reasonable agreement with an energy of $45 \mathrm{meV}$ measured by photoconductivity [15]. The corresponding capture coefficient is $(5.7 \pm 0.6) \times 10^{-5} \mathrm{~cm}^{-3} / \mathrm{s}$, which is comparable to values found by photoconductivity and current-noise studies with boron doping of $5 \times 10^{15} \mathrm{~cm}^{3} / \mathrm{s}$ [16]. Although Fig. 3(b) shows a clear dependence on the magnetic field, it is difficult to extract the dependence of $C_{p}$ and $E_{a}$ separately. However, we have verified that $d C_{p} / d E_{a}>0$ for shallow acceptors (using the analysis of Ref. [17]). Any small magnetic-field-induced change of $E_{a}$ is always towards a higher energy compared to the valence band [11], which evidently corresponds to an increase of $C_{p}$. As a conservative approach no increase in $C_{p}$ is assumed. Thereby, for every reasonable acceptor energy, the fitted acceptor energy consistently shifts with at least $1.8 \mathrm{meV}$ as a function of the magnetic field, causing holes to occupy states with higher energy. Therefore, a larger kinetic energy is required for impact ionization, corresponding to a postponement of the threshold voltage to larger electric field (see Fig. 2). Finally, for thermal energies larger than the field dependent energy splitting, the relative importance thereof decreases, and the MR collapses with temperature, in agreement with the observations of Fig. 3(a).

In the analysis of our data, we have assumed the magnetic field has an impact on the carrier concentration in the bulk silicon, without analyzing the possible role of the $\mathrm{SiO}_{2}$ as well as the spacing between the contacts. Therefore, we prepared samples in which the spacing between the electrodes as well as the interface between silicon and aluminum is varied. First, for larger electrode spacings the threshold voltage in $I-V$ characteristics (not shown) is higher. Although the MR, measured at constant current, is always smaller than the constant voltage MR, it can be accurately determined in a wide range of currents (Fig. 2). The constant current MR increases with electrode spacing, and thus scales with the voltage drop across the silicon [Fig. 4(a)], but seems to saturate around 200\%.

So far, only silicon wafers with native oxides were used for studying the MR. In a second series of measurements the role of the $\mathrm{SiO}_{2}$ was determined. After removal of the native surface oxide layer with a $100 \mathrm{~mol} / \mathrm{m}^{3} \mathrm{HF}$ dip, devices with different interfaces are prepared, namely, with Ohmic contacts, clean Schottky barriers without $\mathrm{SiO}_{2}$, and with a $1.7 \mathrm{~nm}$ and a $3.7 \mathrm{~nm} \mathrm{SiO}_{2}$ layer, where thicknesses were measured by $\mathrm{x}$-ray photoelectron spectroscopy. Ohmic contacts are obtained by annealing the $\mathrm{Al} / \mathrm{Si}$ interface in argon atmosphere at $450^{\circ} \mathrm{C}$ for $30 \mathrm{~min}$, and the $\mathrm{SiO}_{2}$ layer is made by means of plasma oxidation at a pressure of $1 \times 10^{-1} \mathrm{mbar}$ at $15 \mathrm{~W}$. As expected, the devices with a $3.7 \mathrm{~nm}$ tunnel barrier of $\mathrm{SiO}_{2}$ are too resistive for further analysis. The MR of the device with
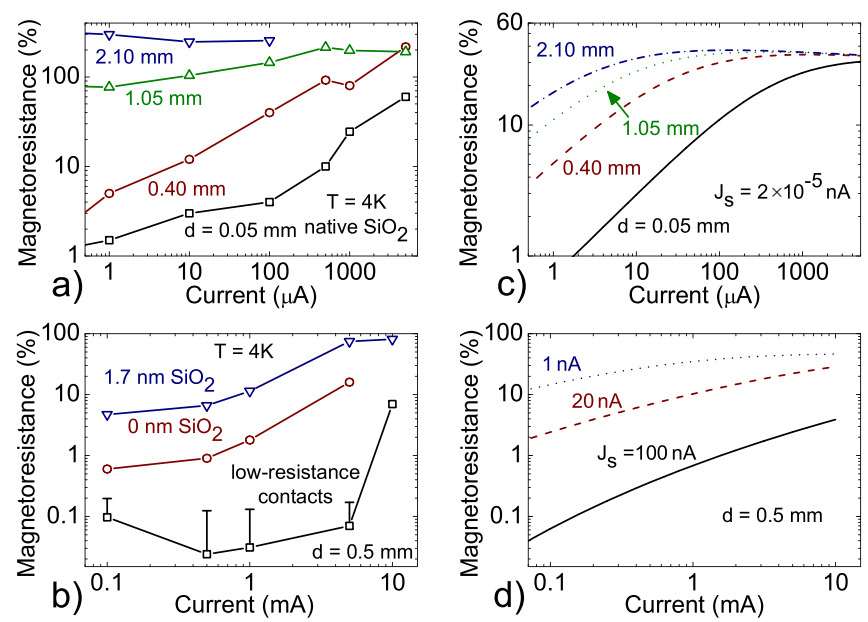

FIG. 4 (color online). Constant current MR for different electrode spacings; (a) measured at $400 \mathrm{kA} / \mathrm{m}$, (c) modeled, and for different contacts; (b) measured at $400 \mathrm{kA} / \mathrm{m}$, (d) modeled.

a $1.7 \mathrm{~nm}$ tunnel barrier of $\mathrm{SiO}_{2}$, which is close to the thickness of the native oxide, is largest [Fig. 4(b)]. With just a Schottky barrier $\left(0 \mathrm{~nm} \mathrm{SiO}_{2}\right)$ only at high currents the MR is $20 \%$, whereas with Ohmic contacts the MR is negligibly small. From $I-V$ characteristics (not shown), it turns out that for high bias voltages the total conductance of a device with wide energy barriers is lower than the total conductance of a device with small energy barriers. This behavior is caused by the lowering of the bulk resistance due to impact ionization of carriers. We conclude that an ultrathin barrier is an indispensable ingredient in creating the large magnitude of this MR effect, since it assists the process of impact ionization.

To clarify these results, a simple model consisting of barrier and bulk contributions to the impact ionization process can be formulated. We speculate that this process is triggered by holes which gain sufficient kinetic energy from the voltage over the oxide $V_{\text {inj }}$ to ionize neutral boron atoms [18]. Additionally, the electric field in silicon must be large enough such that accelerated holes knock other holes free, and the process of impact ionization is sustained.

We consider a balance of the kinetic processes that determine the carrier density $p(E, H)$ at a particular electric and magnetic field. The rate of change of the hole concentration is given by

$$
d p / d t=\left(A_{t}+p A_{i}\right)\left(p_{a}-p\right)-p^{2} C_{p}=0 .
$$

The first term at the right side represents the hole release rate, whereas the second term stands for the rate at which holes are captured by ionized acceptors. Herein, $p_{a}$ is the acceptor concentration. $A_{t}$ is the rate of carrier production by thermal generation and estimated at $2 \times 10^{4} \mathrm{~s}^{-1}$ [19]. $A_{i}$ is the impact ionization coefficient, which is calculated analogously to Cohen and Landsberg [20] as a function of the depth of the acceptor level and drift velocity, which is 
set equal to the hole velocity directly after injection into the silicon, $v=\left(2 e V_{\mathrm{inj}} / m\right)^{1 / 2}$. In accordance with Ref. [20], $A_{i}$ exponentially decreases with the depth of the acceptor level. The current through the barrier in the regime of thermionic emission and tunneling, and the voltage over the silicon are proportional to

$$
\begin{gathered}
J \approx J_{s}\left(e^{\mathrm{eV}_{\mathrm{inj}} / k T}-1\right), \\
V-2 V_{\mathrm{inj}} \approx \frac{1}{p e \mu} \frac{d}{l w} J,
\end{gathered}
$$

with $J_{s}$ the saturation current through the barrier, $\mu$ the mobility, $l$ the thickness of the wafer, $w$ the width of the electrodes, and $d$ the distance between the electrodes. From Eqs. (1)-(3), the total voltage over the device for constant current can be solved for 0 and $400 \mathrm{kA} / \mathrm{m}$ separately. For each current and magnetic field, $p$ is assumed to be constant throughout the silicon. With $J_{s}$ as a fit parameter for each type of $\mathrm{Al} / \mathrm{Si}$ interface, this results in $\mathrm{MR}$ behavior as shown in Figs. 4(c) and 4(d), qualitatively following our experimental data surprisingly well. The data can be interpreted by two conditions that should be fulfilled to obtain a high MR. First, the MR is proportional to $\left(V-2 V_{\text {inj }}\right) / V$ [see the linear increase with electrode spacing, bottom left in Fig. 4(c)], and the largest voltage drop should be over the silicon. Once the voltage over the silicon is dominant, the MR saturates [see Fig. 4(c), at right]. Second, the voltage drop over the oxide layer must be high enough to supply the kinetic energy to the carriers required for the impact ionization process, which is subject to the magnetic field. This condition is fulfilled by either increasing the current or decreasing the saturation current [see Fig. 4(d)], by which $V_{\text {inj }}$, and thus also the MR increases.

Finally, our model shows that such a small magneticfield-induced change of the acceptor level results in a drastic change in the conduction. From a change of the acceptor level energy of $1.8 \mathrm{meV}$ (including a sufficient large voltage to initiate the impact ionization), we deduce a change of around $80 \%$ in impact ionization coefficient (using the analysis of Ref. [20]), explaining the right order of magnitude of maximum constant current MR. However, the extremely large constant voltage MR should be described by an unbalanced interplay between recombination and ionization not covered in Eq. (1), as newly released holes become part of the process. Still, we feel that our transport model captures the most relevant parameters for the large MR in silicon despite the fact that extensions may be required, e.g., to take into account a nonuniform $p$ throughout the silicon. By choosing deeper acceptor levels, we conjecture that this MR effect might be scaled up to room temperature. However, due to a higher effective carrier mass, the energy shift associated with hybridization is much smaller than in $\mathrm{InSb}$ and GaAs, and at room temperature much smaller than the thermal energy. Moreover, the relative change of the impact ionization rate with magnetic field gradually disappears when raising the temperature, thereby precluding an extremely large effect at room temperature.

In conclusion, we observe in boron-doped $\mathrm{Si}_{-} \mathrm{SiO}_{2}-\mathrm{Al}$ structures symmetric positive MR of over $10000 \%$ at magnetic fields around a few hundred $\mathrm{kA} / \mathrm{m}$. Further experimental analysis shows that the transport is dominated by a magnetic-field-controlled process of impact ionization, which is basically understood by introducing a macroscopic transport model.

This work was supported by the Dutch Technology Foundation (STW) via NWO VICI grants.

[1] S. A. Solin, T. Thio, D. R. Hines, and J. J. Heremans, Science 289, 1530 (2000).

[2] F. Y. Yang, K. Liu, K. Hong, D. H. Reich, P. C. Searson, and C. L. Chien, Science 284, 1335 (1999).

[3] B.-C. Min, K. Motohashi, C. Lodder, and R. Jansen, Nat. Mater. 5, 817 (2006).

[4] I. Appelbaum, B. Huang, and D. J. Monsma, Nature (London) 447, 295 (2007).

[5] M. Kobayashi and S. Tanaka, J. Phys. Soc. Jpn. 19, 1246 (1964).

[6] M. Pollak and D. H. Watt, Phys. Rev. 129, 1508 (1963).

[7] S. Witanachchi, H. A. Mourad, H. Srikanth, and P. Mukherjee, Appl. Phys. Lett. 90, 052102 (2007).

[8] S. V. Kravchenko and M. P. Sarachik, Rep. Prog. Phys. 67, 1 (2004).

[9] G. Dearnaley, A. M. Stoneham, and D. V. Morgan, Rep. Prog. Phys. 33, 1129 (1970).

[10] Z. G. Sun, M. Mizuguchi, T. Manago, and H. Akinaga, Appl. Phys. Lett. 85, 5643 (2004).

[11] R. J. Sladek, J. Phys. Chem. Solids 5, 157 (1958).

[12] T. W. Hickmott, Phys. Rev. B 46, 12342 (1992).

[13] C. T. C. M. C. Chen, S. H. Ku, and T. Wang, J. Appl. Phys. 96, 2297 (2004).

[14] D. L. Losee, J. Appl. Phys. 46, 2204 (1975).

[15] J. W. Chen and A. G. Milnes, Annu. Rev. Mater. Sci. 10, 157 (1980).

[16] É.É. Godik and Ya.E. Pokrovskii, Sov. Phys. Solid State 6, 1870 (1965).

[17] W. Schockley and W. T. Read, Phys. Rev. 87, 835 (1952).

[18] P. E. Nicollian, W. R. Hunter, and J. C. Hu, Proceedings of the International Reliability Physics Symposium (IEEE, San Jose, 2000), p. 7.

[19] N. G. Karlykhanov, V. A. Lykov, M.S. Timakova, and M. N. Chizhkov, JETP Lett. 79, 25 (2004).

[20] M.E. Cohen and P. T. Landsberg, Phys. Rev. 154, 683 (1967). 